\title{
How Strong Are Weak Patents?
}

\author{
Joseph Farrell and Carl Shapiro
}

\section{Supplementary Material \\ Licensing Probabilistic Patents to Cournot Oligopolists*}

September 2007

We study here the special case in which downstream competition takes the form of Cournot oligopoly with linear demand and constant marginal cost.

The first (entirely standard) step is to construct the $\pi(a, b)$ and $x(a, b)$ functions. Let downstream demand be $p=A-X$, where $X$ is total output. If downstream firm $i$ produces with constant marginal $c_{i}$, its profits are $\pi^{i}=\left(A-X_{-i}-x_{i}-c_{i}\right) x_{i}$, where $x_{i}$ is its output and $X_{-i}$ is the combined output of all other firms. Firm $i$ 's best-response function is $A-X_{-i}-c_{i}-2 x_{i}=0$ or $A-X-c_{i}-x_{i}=0$. Note that $p-c_{i}=x_{i}$. Adding up for all firms gives $(N+1) X=N A-\sum c_{k}$ and so $(N+1) p=A+\sum c_{k}$. We therefore have $x_{i}=\left(A+c_{-i}-N c_{i}\right) /(N+1)$, where $c_{-i} \equiv \sum_{j \neq i} c_{j}$. Since $p-c_{i}=x_{i}$, we get $(N+1)^{2} \pi_{i}=\left[A+c_{-i}-N c_{i}\right]^{2}$. With $c_{i}=a$ and $c_{j}=b$ for all $j \neq i$,

$$
\pi(a, b)=\frac{(A+(N-1) b-N a)^{2}}{(N+1)^{2}}
$$

and

$$
x(a, b)=\frac{A+(N-1) b-N a}{N+1}
$$

\footnotetext{
* This document is available at Shapiro's web site, http://faculty.haas.berkeley.edu/shapiro. A spreadsheet implementing this example is available upon request from Shapiro, shapiro@haas.berkeley.edu.
} 
We note for future reference that $\pi_{1}(a, b)=-\frac{2 N}{N+1} x(a, b)$ and $\pi_{2}(a, b)=\frac{2(N-1)}{N+1} x(a, b)$. All of these formulas apply if and only if they predict $x(a, b) \geq 0$, which holds if and only if $a \leq(A+(N-1) b) / N$.

In our profit and output notation, we normalized the cost levels so that the cost of producing using the patented technology without any running royalty is zero, and the cost of producing using the backstop technology is $v$. If the cost of producing using the patented technology is $c>0$, c should be added to all cost expressions. This would cause $A$ to be replaced by $A-c$ in all of our expressions, so $A$ should be interpreted as the difference between the intercept on the linear demand curve and the production cost associated with the new technology. Likewise, $A-v$ should be interpreted as the difference between the intercept on the linear demand curve and the production cost associated with the backstop technology.

In thinking about whether $v$ is "small" or "large" in this context, the ratio $v / A$ has meaning, not the absolute level of $v$. The ratio $2 v / A$ approximates the fraction of the overall total welfare that can be generated by this market that is attributable to the patented technology. To see this, observe that without the patented technology, maximum total welfare equals the area under the linear demand curve up to the point where price equals marginal cost under the backstop technology, $v$. This area is $(A-v)^{2} / 2$. With the patented technology, the maximum total welfare is $A^{2} / 2$. The fraction of this total attributable to the patented technology, $\frac{2 v}{A}+\left(\frac{v}{A}\right)^{2}$, is close to $2 v / A$ if $v / A$ is small.

The downstream price is $p(a, b)=\frac{A+(N-1) b+a}{N+1}$. With equal royalty rates, $p(r, r)=\frac{A+N r}{N+1}$ (with constant $p^{\prime}(r)$ as noted in the text), each firm's margin is $p(r, r)-r=\frac{A-r}{N+1}$, $\pi(r, r)=\frac{(A-r)^{2}}{(N+1)^{2}}$ and $x(r, r)=\frac{A-r}{N+1}$. In this case, the patent holder's income from running royalties is $N r x(r, r)$, or $\frac{N}{N+1} r(A-r)$. The combined profits of the patent holder and the downstream firms, measured per downstream firm, are $T(r)=r x(r, r)+\pi(r, r)=r \frac{A-r}{N+1}+\frac{(A-r)^{2}}{(N+1)^{2}}$, or $T(r)=\frac{(A-r)(A+N r)}{(N+1)^{2}}$. Differentiating, $T^{\prime}(r)=\frac{A(N-1)-2 r N}{(N+1)^{2}}$, which is decreasing in $r$. 


\section{Two-Part Tariffs with Downstream Competition}

We assume in the text that $T(r)$ strictly increases in the range $0 \leq r \leq v$. Since $T^{\prime}$ is decreasing in $r$, this will be true if and only if $T^{\prime}(v) \geq 0$, which holds if and only if $v \leq \frac{A(N-1)}{2(N+1)}$. The price/cost margin for a downstream monopolist using the new technology with no royalty burden is $A / 2$, so this condition is satisfied if the size of the patent is no greater than the price/cost margin for a downstream monopolist times $\frac{N-1}{N+1}$. Of course, when $N=1$, due to the inefficiency of double marginalization, $T(r)$ cannot increase with $r$ in the range $0 \leq r \leq v$. But the condition $v \leq \frac{A(N-1)}{2(N+1)}$ is very easily satisfied so long as there is some downstream competition. Even with $N=2$, the condition becomes $v \leq \frac{A}{2} \frac{1}{3}$, which includes moderately large innovations responsible for roughly a third of total available market surplus; and the condition becomes even easier to satisfy as $N$ becomes larger. With $N=4$, it becomes $v \leq \frac{A}{2} \frac{3}{5}$.

We showed in the text that $r(\theta)=v$ if $\theta \leq \theta_{V} \equiv T^{\prime}(v) / \pi_{2}(v, v)$. Substituting, using $T^{\prime}(v)=\frac{A(N-1)-2 v N}{(N+1)^{2}}$ and $\pi_{2}(v, v)=\frac{2(N-1)}{N+1} x(v, v)=\frac{2(N-1)}{N+1} \frac{A-v}{N+1}$, and simplifying, we get

$$
\theta_{V}=\frac{1}{2}-\frac{1}{2}\left[\frac{N+1}{N-1}\right]\left[\frac{v / A}{1-v / A}\right]
$$

If $v / A$ is small, then $\theta_{V} \approx 1 / 2$, and all patents with $\theta<1 / 2$ are licensed using $r(\theta)=v$. As $v / A$ becomes larger, $\theta_{v}$ falls. When $v / A$ reaches its upper limit such that $T^{\prime}(v) \geq 0$, i.e., $\frac{(N-1)}{2(N+1)}, \theta_{V}=\frac{1}{N+3}$. For any given level of $v / A$, as $N$ grows, $\theta_{V}$ rises, approaching $\frac{1}{2}-\frac{1}{2}\left[\frac{v / A}{1-v / A}\right]$. As an example, with $N=5$ and $v / A=1 / 10, \theta_{V}=5 / 12$.

For $\theta>\theta_{v}$, the royalty rate $r(\theta)$ is the solution to $G_{r}(r, \theta)=T^{\prime}(r)-\theta \pi_{2}(v, r)=0$ subject to the constraint $0 \leq r \leq v$. Combining $\pi_{2}(v, r)=\frac{2(N-1)}{N+1} x(v, r)=\frac{2(N-1)}{N+1} \frac{A+(N-1) r-N v}{N+1}$ with $T^{\prime}(r)=\frac{A(N-1)-2 r N}{(N+1)^{2}}$, we can solve $G_{r}(r, \theta)=T^{\prime}(r)-\theta \pi_{2}(v, r)=0$ explicitly for $r(\theta)$, giving:

$$
r(\theta)=\frac{(N-1)(A+2 \theta(v N-A))}{2\left(N+\theta(N-1)^{2}\right)},
$$


so long this expression is non-negative. Since $r(1)=\frac{(N-1)(2 v N-A)}{2\left(N^{2}+N+1\right)}$, so long as $v \geq A / 2 N$, we know that $r(1) \geq 0$, which implies that $r(\theta) \geq 0$ for all $\theta$. If $v<A / 2 N$, then $r(\theta)=0$ for sufficiently strong patents.

However, the derivation of this formula for $r(\theta)$ required that $\pi(v, r) \geq 0$, which is true if and only if $A+(N-1) r-N v \geq 0$, i.e., if and only if $r \geq \frac{v N-A}{N-1} \equiv \hat{r}$. So we need to go back and check that $r(\theta) \geq \hat{r}$. This is equivalent to $\frac{(N-1)(A+2 \theta(v N-A))}{2\left(N+\theta(N-1)^{2}\right)} \geq \frac{v N-A}{N-1}$. Cross-multiplying gives $(N-1)^{2}(A+2 \theta(v N-A)) \geq 2\left(N+\theta(N-1)^{2}\right)(v N-A)$. Simplifying, this becomes $A(N-1)^{2} \geq 2 N(v N-A)$. Expanding and simplifying this expression gives $(A-2 v) N+A \geq 0$. Since $v \leq A / 2$, this inequality holds so the analysis is consistent. The patent holder never finds it optimal to reduce $r$ all the way down to $\hat{r}$; while setting $r=\hat{r}$ would make $\pi(v, r)=0$, at the margin, the lower value of $r$ has no impact on $\pi(v, r)$ but does reduce $T(r)$.

Summarizing, we have $\theta_{V}=\frac{1}{2}-\frac{1}{2}\left[\frac{N+1}{N-1}\right]\left[\frac{v / A}{1-v / A}\right], r(\theta)=v$ for $\theta \leq \theta_{V}$, and $r(\theta)=\frac{(N-1)(A+2 \theta(v N-A))}{2\left(N+\theta(N-1)^{2}\right)}$ for $\theta \geq \theta_{V}$. We now compute the other relevant variables that are generated in this licensing equilibrium.

The fixed fee $F(\theta)$ is given by $\pi(r(\theta), r(\theta))-F(\theta)=\theta \pi(v, r(\theta))+(1-\theta) \pi(0,0)$. Using the expressions already derived for the profit functions, we have

$$
F(\theta)=\frac{(A-r(\theta))^{2}}{(N+1)^{2}}-\theta \frac{(A+(N-1) r(\theta)-N v)^{2}}{(N+1)^{2}}-(1-\theta) \frac{A^{2}}{(N+1)^{2}} .
$$

Next, we have $P(\theta)=N r(\theta) x(r(\theta), r(\theta))+N F(\theta)$ which becomes

$$
P(\theta)=N\left[F(\theta)+r(\theta) \frac{(A-r(\theta))}{(N+1)}\right]
$$

Total welfare at price $p$ and corresponding output $A-p$ is equal to total profits, $p(A-p)$, plus consumer surplus, $\frac{1}{2}(A-p)^{2}$, which equals $\frac{1}{2}(A+p)(A-p)$. The price resulting from royalty rate $r$ is $p(r, r)=\frac{A+N r}{N+1}$. Substituting and simplifying, the total welfare function is

$$
w(r)=\frac{N}{2(N+1)^{2}}[(N+2) A+N r](A-r) .
$$


This can be used to compute $W(\theta)=w(r(\theta))$, as well as $\bar{W}=w(v)-N v x(v, v)$, giving

$$
\bar{W}=\frac{N(N+2)}{2(N+1)^{2}}(A-v)^{2},
$$

which can be used in turn to compute the patent holder contribution,

$$
K(\theta)=w(r(\theta))-[\theta \bar{W}+(1-\theta) w(0)] .
$$

\section{Negative Fixed Fees Not Feasible, Downstream Competition}

We now calculate $s(\theta)$ and $P(\theta)$ using $\pi(s, s)=\theta \pi(v, s)+(1-\theta) \pi(0,0)$.

So long as $x(v, s) \geq 0$, i.e., so long as $v \leq \frac{A+(N-1) s}{N}$, or $s \geq \frac{v N-A}{N-1} \equiv \hat{r}$, the formula $\pi(v, s)=(A-v N+(N-1) s)^{2} /(N+1)^{2}$ applies. (This will be true for all positive $s$ if $v<A / N$, in which case $\hat{r}<0$.) In the range, $s>\hat{r}$, the equation defining $s(\theta)$ is $(A-s)^{2}=\theta(A-v N+(N-1) s)^{2}+(1-\theta) A^{2}$. This expression is quadratic in $s$, so we can solve explicitly for $s$. Writing the quadratic expression in $s$ in the form $\alpha s^{2}+\beta s+\gamma=0$, we have $\alpha=\theta(N-1)^{2}-1, \beta=2 A+2 \theta(N-1)(A-v N)>0$, and $\gamma=\theta v N(v N-2 A)<0$. All of these parameters are linear in $\theta$. In this case, for $v<A / N$ we get $s^{\prime}(0) / v=N-v / 2 A$. As $v \rightarrow 0$ this gives $s^{\prime}(0) / v \rightarrow N$, a special case of the general finding that for small values of $v$ in Cournot oligopoly with constant marginal costs $s^{\prime}(0) / v \approx N$.

However, if $s>\hat{r}$, then $\pi(v, s)=0$, and the equation defining $s(\theta)$ becomes $\pi(s, s)=(1-\theta) \pi(0,0)$. Solving this gives $s(\theta)=A(1-\sqrt{1-\theta})$. If $v>A / N$, define $\hat{\theta}$ by $A(1-\sqrt{1-\hat{\theta}})=\hat{r}$. Solving this gives $\hat{\theta}=1-\left(\frac{N}{N-1}\right)^{2}(1-v / A)^{2}$. Putting the pieces together, we have $s(\theta)=A(1-\sqrt{1-\theta})$ for $\theta \leq \hat{\theta}$ and $s(\theta)$ as the solution to $(A-s)^{2}=\theta(A-v N+(N-1) s)^{2}+(1-\theta) A^{2}$ for $\theta \geq \hat{\theta}$. The resulting $s(\theta)$ applies over the range of patent strengths for which $F(\theta) \leq 0$, or for all patent strengths if licenses are constrained to be linear. If positive fixed fees are feasible, then if $F(\theta)>0$ the per-unit royalty rate equals $r(\theta)$, as already calculated in the section with two-part tariffs, not $s(\theta)$. Once $s(\theta)$ has been calculated, the patent holder's profits and the welfare and contribution functions are easy to derive using $s(\theta)$, exactly as in the case of two-part tariffs where we used $r(\theta)$. 


\section{Vertical Integration}

The patent holder's downstream division behaves just like a firm with zero cost, since changes in its output do not affect the output of the downstream firms and thus the patent holder's royalty income. The formula for profits is $(N+1)^{2} \pi_{i}=\left[A+c_{-i}-N c_{i}\right]^{2}$ if there are $N$ firms in total. We have $N+1$ firms in total, so this becomes $(N+2)^{2} \pi_{i}=\left[A+c_{-i}-(N+1) c_{i}\right]^{2}$ Therefore, putting $c_{1}=0$ for the patent holder, we get $(N+2)^{2} \pi^{I}(a, b)=[A+(N-1) b-(N+1) a]^{2}$. Since $\pi_{1}^{I} / \pi_{2}^{I}=-(N+1) /(N-1)$, this implies that $\rho^{I}=\frac{\left|\pi_{1}^{I}\right|}{\left|\pi_{1}^{I}+\pi_{2}^{I}\right|}=\frac{N+1}{2}$.

\section{Linear Licenses to Downstream Firms That Do Not Compete}

With downstream firms that do not compete, we have a downstream monopolist. Putting $N=1$ into the Cournot equations above, and simplifying the notation, we get $\pi(s)=(A-s)^{2} / 4$, $x(s)=(A-s) / 2$, and $s x(s)=s(A-s) / 2$. The royalty rate $s(\theta)$ solves $(A-s)^{2}=\theta(A-s(1))^{2}+(1-\theta) A^{2}$. The solution to this quadratic equation is $s(\theta)=A-\sqrt{A^{2}-2 \theta A s(1)+\theta s(1)^{2}}$, an increasing convex function of $\theta$. So long as $v<A / 2$, $s(1)=v$, and this equation becomes $s(\theta)=A-\sqrt{\left.A^{2}-2 \theta A v+\theta v^{2}\right)}$, and $s^{\prime}(0)=v\left(1-\frac{v}{2 A}\right)$. 